\title{
Explaining Near-Death Experiences: Physical or Non-Physical Causation?
}

\author{
Robert G. Mays, B.Sc. \\ Suzanne B. Mays, A.A., CMP \\ Chapel Hill, NC
}

\begin{abstract}
A recent comparison of near-death experiences (NDEs) occurring with life-threatening events leading to coma to "NDE-like" experiences occurring with non-life-threatening events showed no significant difference in intensity or content between the two experiences, suggesting that neither the apparent proximity to death nor the specific physiological or psychological factors present influence quality or "depth" of such experiences. This study directly contradicts previous findings of significantly more enhanced perception of light and enhanced cognitive powers in near-death experiencers (NDErs) very close to death compared to those not in danger of dying. We argue that findings of the recent study are more valid due to more appropriate methodology. The general assumption has been that NDEs result from some physical cause that brings people close to death, but such explanations are problematic. An adequate scientific explanation of NDEs needs to be complete, accounting for all aspects of all NDEs. The commonality among NDEs occurring in a wide range of conditions suggests that there is a common underlying "proximate cause" to all NDEs. The proximate cause could be physiological, for example, a common brain circuit in a particular brain region, or non-physiological, for example, the separation of consciousness as an autonomous entity from the physical body. We argue that the latter hypothesis has greater explanatory power, although it posits elements beyond the current physicalist paradigm. Which explanation of NDEs is correct will be resolved only with further scientific investigation.
\end{abstract}

KEY WORDS: near-death experience depth; near-death experience causes; selfconscious mind; mind-separation hypothesis; mind-brain interaction.

Robert G. Mays, B.Sc., is a retired senior software engineer, and Suzanne B. Mays, A.A., is a Certified Music Practitioner (through the Music for Healing and Transition Program) who provides palliative care to hospitalized patients. They have studied near-death phenomena together for more than 35 years. Reprint requests should be addressed to Mr. Mays at 5622 Brisbane Drive, Chapel Hill, NC 27514; e-mail: mays@ ieee.org. 
A fundamental question in the field of near-death studies is the mechanism underlying near-death experiences (NDEs): What "causes" them? The answer may be informed in part by the answer to a more specific question: What is the relationship between closeness to death and other conditions of NDEs and the depth and content of NDEs? In this paper we will first summarize a recent study in which researchers addressed the latter question, then contextualize their findings in light of previous research on this topic, and finally discuss the implications of their findings for the question of a common proximate cause of NDEs. We conclude by examining possible physical and non-physical causes of NDEs.

\section{Does Proximity to Death Determine the "Depth" of a Near-Death Experience?}

\section{Comparison of a Recent and a Previous Study}

Recently, researchers from the University of Liège, Belgium, led by Vanessa Charland-Verville and Steven Laureys (2014), with assistance from members of the International Association for Near-Death Studies (IANDS) in France and Belgium, published a report in Frontiers in Human Neuroscience. Their study compared NDE reports resulting from life-threatening events leading to coma (which they termed "real NDEs", $n=140$ ) to "NDE-like experiences" occurring after non-life-threatening events $(n=50)$, such as during sleep $(n=13)$, fainting $(n=11)$, meditation $(n=5)$, drug or alcohol use $(n=3)$, or other non-life-threatening situations $(n=18)$. Subjects were included in the study who scored 7 or higher out of a possible 32 on the NearDeath Experience Scale (NDE Scale; Greyson, 1983). Higher scores indicated relatively greater intensity, and greater number of features or elements indicated relatively richer content.

Surprisingly, the results showed no significant difference in either the intensity or content of the NDE between the "NDE-like" and "real NDE" groups. Furthermore, there was no difference in the "real NDE" group depending on the cause of the coma-anoxic condition resulting from cardiac arrest or drowning $(n=45)$ versus traumatic injury $(n=30)$ versus other cause of coma such as illness or surgical complications $(n=65)$.

This finding means that neither the apparent proximity to death nor the specific physiological or psychological factors that were present at the time of the NDE influenced the intensity or content of the NDE. A person, for example, who has an "NDE-like" experience dur- 
ing sleep or meditation will tend to have the same features (e.g., feelings of peace, separation from the body, a brilliant light) and the same intensity (total NDE score: mean of 16 for "real NDEs" and 17 for "NDE-like") as a person actually near death who has a so-called "real NDE," for example while suffering anoxia and coma from cardiac arrest. NDEs occurring at either end of the closeness-to-death spectrum cannot be distinguished: They are the same experience. These results imply that there is no physiological explanation that can account for all NDEs. Given that many of the "NDE-like" experiences occurred in non-life-threatening situations with no threat of physiological harm, such as during sleep and meditation, the explanation that such NDEs are triggered by the strong belief or fear of dying also does not hold. The researchers concluded,

it seems that NDEs cannot be explained solely by the closeness to death or by the etiology of the precipitating factor. The question whether the NDEs' extraordinary features can be fully explained by cerebral activity is still a matter of debate.... (Charland-Verville, Jourdan, Thonnard, Ledoux, Donneau, Quertemont, \& Laureys, 2014, p. 6)

Charland-Verville et al. (2014) did not address the apparent contradiction between their results and the oft-cited analysis by Owens, Cook, and Stevenson (1990). In the earlier study, NDErs whose records confirmed had been medically so close to death that they would have died without medical intervention $(n=28)$ reported significantly more enhanced perception of light and enhanced cognitive powers than did NDErs whose medical records showed they were not in danger of dying even though most of them thought they were $(n=30)$. Owens et al. (1990) further observed, "It would seem that among those [persons] who were not near death, their experiences were precipitated by their belief that they were" (p. 1177).

The results of the 1990 analysis - that NDEs have more features, in particular enhanced light and enhanced mentation, in subjects who were closer to death-have been cited frequently (for example, Carter, 2010, p. 126; Greyson, 2000; Greyson, 2010a, p. 40; Zingrone \& Alvarado, 2009 , pp. 26,38 ) to infer that there is a greater "depth" to the experience with greater proximity to death. The further inference is that when an NDE occurs in a non-life-threatening circumstance, there most likely is a perceived threat of imminent death and that psychological fear is the trigger for the experience. These results and inferences have become accepted as general facts about NDEs among many NDE researchers. The results of Charland-Verville et al. (2014) challenge those conclusions. 
There are three major methodological differences between the two studies. The 1990 study (a) used an ad hoc classification of NDE features rather than the NDE Scale and included features that are not included in that Scale (e.g., experience of a tunnel), (b) combined several NDE features as a generic "enhanced cognitive function" (e.g., speed, logic and clarity of thoughts, control of cognition, and overall clarity and vividness of senses), and (c) compared two NDE groups based on the severity of the medical condition - those who would have died without medical intervention and those whose medical records showed they were not actually in danger of dying-rather than the recent study's criteria of the medical extremes of coma and truly nonlife-threatening situations.

These methodological differences alone probably do not explain the difference in the final result. Rather, the differing results may also reflect variations in sampling and other aspects of methodology. Presumably the features of the 1990 not-near-death experiences would be similar to those of the 2014 NDE-like experiences, but they were notably different. Enhanced light was reported by $40 \%$ (12 of 30) of the 1990 sample compared to 84\% (42 of 50) of the 2014 sample. Enhanced cognition was reported by 50\% (13 of 26) of the 1990 sample compared to $68 \%$ (34 of 50) of the 2014 sample. (Indeed, because the 1990 study combined several criteria for "enhanced cognitive function," there is no easy comparison between the studies without the specific data. It is very likely that more than $68 \%$ of the 50 "NDE-like" subjects in the recent study would be judged to have experienced "enhanced cognitive function" when all of the relevant NDE scale features are combined. The $68 \%$ in the recent study represents only reports of "more vivid senses.") Although statistical comparison of these results is not possible, the differences appear to be substantial.

These differences in results are probably due to inadequate statistical sampling in one or both studies or possibly incomplete NDE narratives in the 1990 study because an established assessment instrument was not used. The recent study is methodologically superior to the previous study in several respects: (a) sample size: 190 versus $58 \mathrm{NDE}$ reports, respectively; (b) instrumentation: the use of an established, psychometrically sound instrument to assess NDE intensity and content in the recent but not previous study; and (c) range of physiological and psychological conditions covered: The recent study included 50 reports with truly non-life-threatening events, whereas the previous study included only cases with some level of adverse medical condition. 
Given the wide discrepancy between the two results, further research is warranted: a replication of the recent study or at least further study of non-life-threatening NDEs, which have not received much attention and analysis prior to the recent study.

\section{Methodological Clarity}

The discrepancy between the findings of Owens et al. (1990) and Charland-Verville et al. (2014) highlights the difficulties in comparing individual NDE features in subsets of NDE cases, such as "near death" versus "not near death." NDEs are complex narratives containing many details that describe numerous features, various affective states, and profound transcendental or mystical aspects. By isolating individual features or collections of features, such as "enhanced cognitive functions," investigators risk introducing biases, not only in potentially missing the feature when the feature was present in the NDE but not explicitly stated in the narrative, but also in coloring the result with implicit value judgments of which features constitute greater "depth," for example, experiences of profound light versus profound darkness.

We suggest that the comparison of NDEs based on individual NDE features is inherently problematic. It would be better to restrict the comparison of subsets of NDEs to the measures used by CharlandVerville et al. (2014), namely the intensity of the NDE based on the total NDE Scale score and the overall content of the NDE based on the total number of NDE features reported on the NDE Scale. The use of the NDE Scale can provide continuity of measurement across studies.

It is worth noting that Surbhi Khanna and Bruce Greyson (2014) recently defined the "depth" of an NDE more precisely by the numerical NDE Scale score: 0-6 as "no NDE," 7-14 as "subtle," 15-22 as "deep," and 23-32 as "profound". The criterion for these categories is based on the deviation of the NDE Scale score from the mean score of 15 for the 74 NDEs whose scores went into defining the Scale: "subtle" NDEs fall within one standard deviation below the mean; "deep" NDEs fall within one standard deviation above the mean; and "profound" NDEs are more than one standard deviation above the mean. In effect, Khanna and Greyson (2014) have equated the depth of an NDE with the aggregate intensity of its NDE Scale features. Although Charland-Verville et al. (2014), by virtue of publishing their results the same year as Khanna and Greyson (2014), did not have the benefit 
of the latter's findings regarding specific categorization of depth, their use of the continuous score as an indication of intensity reflected a similar approach that lends further credibility to their results.

\section{Multiple Antecedent Causes versus a Common Proximate Cause of NDEs}

The results of the study by Charland-Verville et al. (2014) strongly suggest that NDEs occur completely independent of NDErs' specific antecedent physiological or psychological conditions and, in some cases, appear to occur spontaneously, that is, with no apparent antecedent condition. It follows, then, that there is no single physiological or psychological explanation for NDEs. Rather, NDEs appear to be a characteristic altered state of consciousness that may be facilitated or triggered by many different types of antecedent conditions or may have no apparent facilitating condition or event.

In seeking for an explanation of the cause of NDEs, theorists need to consider what constitutes an adequate scientific explanation. In their recent critique of Pim van Lommel's (2013) theory of non-local consciousness, Benjamin Mitchell-Yellin and John Fischer (2014, p. 174) stated that an adequate scientific explanation of NDEs needs to be complete; that is, it should account for all aspects of all NDEs. They qualified this requirement with the stipulation that there may be contexts in which an explanation of a phenomenon is adequate even though it does not explain all aspects of every instance of the phenomenon.

We agree with Mitchell-Yellin and Fischer: An adequate scientific explanation of the cause of NDEs needs to account for all cases, or nearly all cases, of NDEs. The explanation also needs to account for cases in which the cause is present but no NDE occurs. For example, hypoxia is commonly cited as the cause of NDEs, but many NDEs occur in the absence of hypoxia, so hypoxia alone is not an adequate explanation of the cause of NDEs. Indeed, if NDEs can occur in cases with no identifiable, unique physiological or psychological antecedent, but with equal intensity and content as NDEs occurring in the antecedent condition of clinical death, then the cause of NDEs per se can't be any particular known physiological or psychological condition.

The commonality of intensity and content in NDEs-feeling separated from the body, seeing or feeling surrounded by a brilliant light, entering an unearthly world, and so on-under a wide range of con- 
ditions suggests that a common state of consciousness occurs during NDEs. The common state of consciousness suggests that there is a common proximate or immediate cause of the experience. There may be any number of antecedent conditions that lead to or trigger a common precipitating cause-a common mechanism - that results in the NDE. An antecedent condition may occur-for example, a cardiac arrest-but if the proximate cause is absent, no NDE occurs, as happens for more than $80 \%$ of cardiac arrest survivors. Conversely, the proximate cause may occur in the absence of a known antecedent condition, resulting in an NDE, as is the case with people who are not near death but who have experiences indistinguishable in content and intensity from experiences that occur in near-death circumstances.

The proximate cause could be a physiological condition that occurs in both near-death and not-near-death circumstances, or it could be some non-physical process. In either case, the explanation based on it would need to account for NDEs occurring in a variety of conditions, including ordinary conditions, like sleep, meditation, or glancing at a sunrise, and would thus satisfy the requirement that it explain all NDEs. It also should account for how, under seemingly identical antecedent conditions - such as two people in cardiac arrest, two people meditating, or even the same person in cardiac arrest or meditating on two different occasions-an NDE does occur in one case and not the other. Furthermore, the explanation should account more broadly for all aspects of all NDEs.

\section{Possible Physiological or Psychological Proximate Causes of NDEs}

Although there doesn't appear to be an obvious physiological or psychological condition that acts in all cases of NDEs, physiological or psychological explanations should nevertheless be considered fully. In explaining spontaneous out-of-body experiences (OBEs), psychiatrists Glen Gabbard and Stuart Twemlow (1984) proposed that in certain altered states of consciousness, "undercontrol of the usual sensory and perceptual processes . . results in the unplanned emergence of images" (pp. 238-239). They postulated that these images take over when external sensory input is quieted, resulting in fabrications that are taken as real and interpreted as an OBE, that is, the uncoupling of the bodily ego and mental ego. Gabbard and Twemlow (1984) proposed that an $\mathrm{OBE}$ is the "final common pathway of many different 
causative factors" (p. 239), whereby the uncoupling may result from any number of stimuli including a variety of unconscious psychological issues, hallucinogens, or merely decreased proprioceptive signals. Their ego-uncoupling model is integrative rather than pathological, allowing for the highly complex perceptual and cognitive functions that are reported in OBEs and, by extension, in NDEs.

Another possible proximate cause with a physiological origin was proposed recently by Enrico Facco and Christian Agrillo (2012). In their view, NDEs may be caused by a physiological agent that triggers a common brain circuit in a particular brain region, such as the temporal lobe or right angular gyrus, or by an agent that works like a psychotropic drug by affecting particular neural receptors, or by some combination of these mechanisms. Facco and Agrillo (2012) proposed that the agent activates "in life-threatening situations (both organically and psychologically), in psychiatric and neurological disorders, but also in hypnosis and meditation, role transitions and, more generally, in all deep existential crises" (p. 5) to induce the altered state of consciousness of an NDE. Facco and Agrillo (2012) did not elaborate their hypothesis any further or provide an explanation of how specific features of NDEs could arise from their model.

\section{Deficiencies of Physiological and Psychological Explanations of NDEs}

Psychological explanations such as that offered by Gabbard and Twemlow (1984) and physiological explanations such as that offered by Facco and Agrillo (2012) are plausible, but they appear to suffer several deficiencies. The first deficiency is that a number of NDEs appear to have occurred when the subject was clinically dead, often for more than 10 minutes. By current scientific understanding, in these cases there could have been no brain function to support any conscious experience, let alone an experience that included hyperreal perceptions, lucid thinking, and vivid memory formation. The timing of these NDE cases can be established by verification of "apparently non-physical veridical perceptions" (AVPs; Holden, 2009) in which NDE perceptions that should have been impossible based on known physical processes were nevertheless subsequently confirmed accurate. In several of these cases, the perceptions were purely visual-so there was no possibility of unconscious auditory or tactile impressions-and the patient's eyes were taped shut. For recent examples, see Titus Rivas and 
Rudolf Smit (2013) and a case of AVP from the AWARE study (Parnia et al., 2014).

In these cases, it is hard to justify that a physiological or psychological brain mechanism could be operant, because the brain-by all current means of assessment-is not functioning. A physical explanation would need to show how the brain was in fact functioning at the time in some way, for example with unusual sensory capability, or that the content of the NDE was constructed from other sources (or the patient's unconscious) after the patient regained normal brain function.

Indeed, Mitchell-Yellin and Fischer proposed such physical explanations in analyzing two cases of AVP that they cited from van Lommel (2013) — the dentures case and the Pam Reynolds case. They further cited Janice Holden's (2009) statement that "in a few of these strongly evidential cases [of AVP], most investigators have ruled out alternate explanations to the hypothesis of nonphysical perception. Nevertheless, because of the uncontrolled nature of anecdotal cases, alternate explanations remain open to debate" (p. 210). They argued that rejection of a physical explanation of NDEs is premature and that controlled investigation of AVPs is warranted-a process that NDE researchers have been pursuing since the 1980s.

Holden's statement was very reasonable at the time of her research, first presented in 2006. In the meantime, a number of additional cases of corroborated AVPs have been documented (Rivas, Dirven \& Smit, 2013), so the argument by Mitchell-Yellin and Fischer that such experiences could have been generated by physical, sensory means-either at the time of the perceived events or later, after the brain came back on-line-is much weaker than it may seem.

A second deficiency of physiological and psychological explanations of NDEs is the explanation for another feature of AVP: veridical perceptions from a vantage point that was impossible for the patient to observe at the time of the event or at any time during the patient's medical care. For example, during an NDE the patient observes an event, such as hearing a conversation or seeing unusual behavior, at a distance far removed from the patient, for example on a different floor of the hospital. Or the patient observes a unique object out of normal physical view-a 1985 quarter located on an 8-foot high cardiac monitor or the 12-digit serial number located on top of a 7-foot high ventilator machine-which was later verified by others (Rivas, Dirven \& Smit, 2013). In these cases, the patient may not be clinically dead during the NDE but they report an AVP perceived from a vantage point 
outside of their physical body and impossible to perceive by ordinary means because of the physical spatial separation. Moreover, in cases of observing a specific event, the time of the event itself establishes the time of the NDE.

Again, in these cases, it is hard to justify that a physiological or psychological brain mechanism could be operant. In their critique of van Lommel's theory, Mitchell-Yellin and Fischer did not address cases of this type, although van Lommel (2013) included them as phenomena covered by his theory, citing "many corroborated cases of veridical perception from a position out of and above the body during NDE" (p. 20) and that "even blind people have described veridical perceptions during out-of-body experiences at the time of their NDE" (p. 22). It is conceivable that such experiences are generated by some physical, sensory means, either at the time of the perceived events or later after the patient recovered from the incident. However, an unusual physiological sensory ability would be needed to accurately report events at far distances or to accurately describe unusual objects observed out of the physical line of sight-an ability so unusual as to be dubious.

A third deficiency of physiological and psychological explanations of NDEs is the explanation for yet another form of AVP: cases of receiving specific previously unknown information during an NDE. There are several different types of cases of this sort. For example, the NDEr may see a deceased person whom they did not know had died. Or the NDEr may meet a deceased person whom they do not know but later recognize as a deceased relative from a photograph. Or the NDEr may be shown a preview or flash-forward of their life that later comes to pass (Greyson, 2010b; Rivas, Dirven \& Smit, 2013; van Lommel, 2010, pp. 32-33, 38). In all of these cases, the patient may not be clinically dead during their NDE but they report transcendental experiences in which they received information that is later verified to be accurate. The information may be simply factual knowledge, but frequently the NDEr sees an image of the person or a picture of the future event, with details of appearance, clothing, conversations and gestures, all of which are later verified.

In these cases as well, it is hard to justify a physiological or psychological brain mechanism. In their critique of van Lommel's theory, Mitchell-Yellin and Fischer also did not address cases of this type, although van Lommel (2013) included them as phenomena covered by his theory, mentioning the life preview in which future images from personal life events are seen, and including a specific case of meeting a deceased person unknown at the time-an NDEr saw a man unknown 
to him looking at him lovingly whom he recognized 10 years later from a photograph as his deceased biological father (p. 21). It is conceivable that such experiences are generated by some physical means such as naturally occurring hallucinogenic processes. However, the fact that the information received is accurate makes such explanations appear very improbable. Furthermore, unlike hallucinations, which tend to be disorganized and devoid of real-world relevance, the information from such NDEs is coherent and personally relevant to the NDEr.

A fourth deficiency of physiological and psychological explanations is the proposal that the NDE content is imagined or is fabricated from subliminally perceived impressions just before or immediately after the period of unconsciousness, or by confabulation with subsequently acquired information. Such explanations do not fit the actual characteristics of NDE memories. A study led by Marie Thonnard and Stephen Laureys (2013) compared the memories of NDEs with memories of others who were in coma without an NDE. The researchers found that memories of NDEs are significantly different from those of coma patients without an NDE. In particular, NDErs' memories have significantly more characteristics, like visual details, memory clarity, self-referential information (being involved in the event), and emotional content. The researchers proposed that NDEs can't be considered as imagined events, which have significantly fewer characteristics. The events in NDEs are more accurately understood as events that NDErs actually perceived and in which the NDErs remember having been actively involved.

In this case as well, it is hard to justify a physiological or psychological mechanism that can account for the nature of NDErs' memoriesclear, hyperreal perceptions and active involvement-such as attributing them to conditions of "undercontrol of the usual sensory and perceptual processes" as Gabbard and Twemlow (1984, p. 238) proposed. Such a mechanism would need to account for how imagined or fabricated memories can be formed via physiological or psychological means such that they take on the quality of memories of actual perceptions and actual involvement in events where, purportedly, no perceptions or involvement actually occurred.

\section{A Possible Non-Physical Proximate Cause of NDEs}

In earlier papers, we have proposed another possible proximate cause of NDEs that is independent of physiology and psychology, based on the view that the human being consists of a nonmaterial "mind" that 
is spatially coextensive and usually intimately integrated with the physical body (Mays \& Mays, 2008; 2011) during life. J. Kenneth Arnette's $(1992,1995,1999)$ theory of essence is another exposition of this idea. The mind or "essence" of a person is an objective, autonomous entity, a "field of consciousness," that interacts energetically with the brain's neural electrical activity to mediate all cognitive faculties. The mind is the seat of consciousness of the person, the subject in which phenomenal experience occurs. Ordinarily, the electrical interaction between the brain and the mind is required for phenomenal experience and consciousness.

In this view, a person's mind entity can, under various conditions, separate from and operate independent of the physical body. There are thus two states of consciousness: an "in-body" state, whereby the mind is united with the brain and body and is dependent on the brain for normal cognitive functions (perception, thought, volition, memory, etc.), and an "out-of-body" state whereby the mind is separated from the brain and body and can function completely independent of them. In the separated state, there is no brain interaction; thus, visual, auditory and other sensations occur directly in the mind without the physical sensory apparatus of the brain. Other cognitive functions also operate purely in the mind entity, such as the ability both to see and see through "solid" objects.

The mind-entity theory is derived from the phenomenology of NDEs, in particular the out-of-body component of NDEs (Mays \& Mays, 2008): the sense of separation from the physical body, hyperreal perceptions and lucid thoughts, veridical perceptions from a vantage point separated from the body, absence of physical pain, absence of pre-existing physical disabilities including the ability to see in congenitally blind NDErs, the sense of returning to the body with subsequent return of pain and disabilities, vivid indelible memories of the experience, and so on. The phenomenon of apparent separation of the NDEr's consciousness in the NDE is a coherent and self-consistent experience that is totally consistent with the idea that consciousness (mind) is an independent entity.

The mind is nonmaterial-does not consist of material atoms, etc. -but rather appears to be a structured, energetic region of space that can interact with physical processes, in particular with neurons, and thus has physical attributes. The mind is united and co-extensive with the brain and body and interacts directly with the brain, probably via electrical interactions with dendritic cortical structures. In this sense, 
our theory is both physical, in that there is direct physical interaction, and non-physical, that is, nonmaterial.

The mind is also the seat of phenomenal experience, that is, a "field of consciousness." All cognitive faculties-perception, thinking, feelings, volition, memory and self-awareness-reside in the nonmaterial mind entity, not in the brain. However, the mind ordinarily is completely dependent on the brain's neural activity for consciousness. Mental events become conscious only when there is sufficient electrical brain activity. On the other hand, the mind can initiate electrical brain activity and thereby serves as the agent that initiates volitional activity, directs attention, exerts "mental force," and alters brain neural patterns plastically. When brain structures are damaged or impaired with drugs, the sensory, motor, and cognitive faculties dependent on them are partially or totally impaired.

The qualitative character of phenomenal experience, for example, of redness, is dependent on the mind's interaction with specific brain electrical activity in a particular part of the cortex. The brain has a complex physical structure that is reflected in both distinctive cognitive functional areas and distinctive cell structures. Because the mind needs to selectively interact in close proximity with specific neural structures, the mind must have an equally complex internal structure that corresponds closely with the brain's physical structure. In fact, it is likely that the internal structure of the mind's field directly maps to the neural structure throughout the cortex.

In this view, the separation of the mind from the physical body is the proximate cause of NDEs. Various physiological and psychological conditions can trigger the separation of the person's conscious entity from the body, or the separation can occur spontaneously, that is, with no apparent antecedent physiological or psychological condition.

\section{Evidence for the Objective Reality of the Mind}

The evidence for the objective reality of the mind as a field of consciousness that interacts energetically with the brain comes primarily from NDEs and related phenomena. Several aspects of NDEs suggest that the mind is objectively real.

First, the numerous reports of AVPs during NDEs from a vantage point outside of physical body, many occurring while the NDEr is clinically dead (Rivas, Dirven \& Smit, 2013), suggest that the locus of conscious awareness can separate from and operate independent of the 
physical body and brain processes. As we described earlier, in many of these cases the perceptions were purely visual, and the patient's eyes were taped shut. In other cases, veridical perceptions were reported from a vantage point that was impossible for the patient to observe by ordinary means, either a unique event observed at a distance far removed from the patient or a unique object impossible to perceive by ordinary means because of physical spatial separation.

Second, the experience in nearly all NDEs is one of hyperreal perceptions, lucid thinking processes, and indelible memory formation, in many cases while the NDEr was under anesthesia, in a coma, or clinically dead. The heightened state of consciousness while the brain is physically dysfunctional or, by all known means of assessment, nonfunctional suggests that consciousness during an NDE operates independent of the brain.

Third, NDErs frequently report a sense of separating from the physical body, hovering over the body with accurate perceptions of the body being resuscitated, and ultimately returning to and reuniting with the body. For most NDErs their physical body appears to be an empty shell. These experiences are consistent with an actual separation of the locus of consciousness from the physical body.

Fourth, NDErs frequently report that the sense of one's consciousness leaving and/or functioning apart from the physical body is accompanied by a sense of being released from the constraints of the physical body - no pain, no physical disabilities, with vivid senses and thoughts. The constraints-pain, physical disabilities, with dulled senses and thoughts-return when the NDEr subjectively experiences returning to the body. These experiences are consistent with separation from the physical body, including from brain processes and bodily functions.

Fifth, some NDErs report seeing their own luminous "body," either with arms and legs or as an ovoid form. In cases where several people have an NDE together (a "group" NDE), the NDErs see one another in an out-of-body state as a luminous body. In a few cases, the NDEr appears as an apparition to one or more other persons at a distance seemingly during the time the NDEr was having the NDE. The NDEr reports having visited the other person, while the other person reports having seen the NDEr at that time (see Rivas, Dirven \& Smit, 2013, chapter 7). All of these reports are consistent with the NDEr's mind having an energetic bodily form that has an objective reality located in space and is in some cases observable by others. 


\section{Evidence for the Interaction of the Mind with Physical Processes}

The phenomena of NDEs suggest that the mind is a nonmaterial entity that separates from the physical body, has subtle interactions with physical processes in the separated state, and then reunites with the physical body. The aftereffects of NDEs include unusual electrical phenomena, which suggests that the interactions of the mind entity are energetic and electrical in nature.

However, in general there is no apparent interaction between the NDEr's energetic body and physical objects. The energetic body appears to be completely nonmaterial and does not appear to interact with material objects because it passes easily through them. The NDEr cannot be heard when speaking and is invisible to ordinary sight. Nevertheless, the NDE literature includes a number of reports of NDErs having subtle interactions in different modalities with physical processes (Mays \& Mays, 2008; 2011).

First, there is apparent interaction with physical processes such as light and sound, because the NDEr reports veridical visual and auditory perceptions. There also is apparent interaction with material objects, because the NDEr can sometimes feel slight resistance when passing through objects such as walls, can bob on the ceiling and feel the support of the hospital roof, and can "touch" and feel a material object. There is one account of an NDE involving apparent interaction of the NDEr's "body" with fog on a cold night. The NDEr jumped up and down and the "jumping fog" was seen by another man who reported it to the police.

Second, the NDEr's body appears to be luminous in some way. We have already mentioned cases in which the NDEr reported having seen one's own luminous energetic body, cases of "group" NDEs in which several NDErs reported having seen the luminous forms of the other NDErs, and cases of the NDEr reportedly having been seen as an apparition by others located at a distance. In addition, the NDEr's energetic body can apparently be "seen" by animals, in one case by a dog.

Third, the NDEr's energetic body can apparently interact with another person's body: An NDEr's hand went through the doctor's arm, which felt "gelatinous" with an electric current; and an NDEr could tickle the nose of another patient, and she would sneeze. In at least four cases, an NDEr reportedly "merged" with another person and was able to see and feel what the other was seeing, feeling, and think- 
ing. All of these cases imply that it is possible for the energetic body to interact with and influence neural activity in the brain; in particular, "merging" implies that the mind/energetic body readily joins with and interacts with the brain, sometimes even another person's brain.

All of these cases suggest that the mind entity, while separated from the physical body, can interact with physical processes even though the mind appears to be nonmaterial. The interactions appear to be subtle but subjectively perceptible to the NDEr and in some cases by an in-body person. Therefore, it is possible that the nonmaterial mind entity can interact with the neurons in the brain and body.

\section{A Possible Mechanism for Mind-Brain Interaction}

The possibility that the mind entity can interact with neurons is not enough. A complete theory of mind-brain interaction needs to have a plausible mechanism for interactions that makes sense both physiologically at the neuron level and neurologically at the level of brain function (Mays \& Mays, 2011).

There is a high correlation of conscious awareness with cortical neural activity. Numerous functional networks have been identified in the cortex, which are active in various behavioral situations, in the resting state, and at various levels of consciousness (Raichle \& Snyder, 2007; Smith et al., 2009; Thibaut et al., 2012). The different regions of the cortex have different broad functions: sensation, recognition, motor action, attention, memory, and executive functions.

These cortical regions must interface with the mind to produce different subjective results, including perceptual processes, volitional motor planning and action, focus of attention, mental deliberation, and memory formation and recall. For the mind to have these different interfaces, it must be similarly "structured" to engage each of the different brain regions.

Because of the high correlation of consciousness with cortical activity, the interface of the mind with the brain must be in the cortex, specifically where the electrical activity is-in the gray matter at the cortical surface. The neurons in the gray matter occur in six layers in the outer $2.5 \mathrm{~mm}$ of the cortical surface. Neural processes called apical dendrites project vertically from cell bodies in the lower layers to the top Layer 1 . We propose that the interface between the mind and brain occurs in these apical dendrites.

Given what is known of the interconnections of the gray matter cells, we propose that the Layer $2-3$ cells serve as the brain-to-mind or sen- 
sory interface and that the Layer 5 cells serve as the mind-to-brain or volitional and attentional interface. Specifically, the electrical pulses from action potentials in the Layer 2-3 cells propagate backwards into the apical dendrites and are "sensed" by the mind. Similarly, the mind initiates action potentials in the Layer 5 neurons, again via the apical dendrites. We suspect that the mechanism of the mind's interaction causes specialized ion channels in the apical dendrites to open, generating action potentials. We believe that the interaction of the field of the mind with neurons can be demonstrated empirically.

Thus, the correct understanding of the brain is no longer an organ that produces consciousness but an organ that interfaces between the body and the conscious mind. In this view, the experience of consciousness in a living person should be considered the result of the interaction of neurological processes in the brain with processes in the nonmaterial mind, the actual seat of a person's consciousness. Together, the mind and brain produce conscious awareness and all cognitive functions in a living person.

In a living person, the mind is completely dependent on the brain to become conscious. The work of Benjamin Libet (2004) has shown that mental events become conscious only when there is sufficient electrical brain activity, generally a third to a half second. If the electrical activity is not sufficient in strength or duration, the percept or other mental event remains subliminal. Libet proposed that this principle of minimum electrical activity applies to all awareness, including awareness of one's thoughts, decisions, and memories. Libet's observations are consistent with the experience of NDErs that the brain seems to provide resistance to consciousness, compared with their experience during the NDE of hyperreal perceptions, lucid thoughts, extra-physical abilities, and indelible memory formation while outside their bodies.

\section{Explanatory Power}

A theory that posits elements, such as a nonmaterial mind entity, which do not fit the currently accepted physicalist paradigm, needs to provide greater explanatory power than the physicalist view of reality. We propose that this theory explains some of the major enigmas of consciousness and the brain.

The first enigma is also called the "hard problem" of consciousness (Chalmers, 1996; 2010), namely, how do action potentials in brain neurons turn into subjective phenomenal experience: the rosy pink of a sunrise, the soft coolness of a morning breeze, the sharp smell of 
fresh pine, or the soft chirping of birds? Neuroscience explains that sensory impressions are turned into neural impulses or action potentials in the different sensory regions of the brain. How does the firing of action potentials that are indistinguishable in these cortical regions produce these different phenomenal qualities?

In the present theory, phenomenal experience occurs from the interaction of cortical action potentials with the mind entity, which is the seat of consciousness. The different qualities or "qualia" arise because of the spatial location of the sensory neurons relative to the mind's own "structure."

The second enigma is also called the "binding problem" (Bayne \& Chalmers, 2003), namely, how do the qualities of phenomenal experience seem to come together in a complete, seamless unity of experience from different, widely separated regions of the brain? How do the rosy colors of the sunrise, the coolness of the breeze, the smell of the pine. and the chirping of the birds form a unified experience as one stands outside viewing the sunrise? If the neural action potentials occur in spatially separated sensory brain regions, where and how do they combine to form this seamless unity of experience?

In the present theory, phenomenal experience appears unified because the mind as the seat of consciousness is unitary. The disparate elements of phenomenal experience are unified within the mind, resulting in the seamless unity of consciousness. The action potentials in different parts of the cortex come to consciousness within the mind and are integrated through purely mental processes into a unified phenomenal experience.

The third enigma is the apparent encoding of declarative memory throughout the cortex. The hippocampus is the critical cortical structure for the formation, consolidation, and recall of episodic memories. Other declarative memories - of facts, word meanings, faces, etc.are formed and recalled through other brain interfaces with the mind. How do the neural circuits work, not only to provide the specific functions of a particular cortical area but also to provide the mechanism for encoding and recalling memories?

In the present theory, memory is formed in the mind entity. The memories of an NDEr's experience are typically vivid and indelible despite the NDEr being - in the most extreme cases-clinically dead. The NDEr typically will say that the memory of the experience is as vivid as when it happened; one can remember-and even reliveevery detail. In ordinary consciousness, memories are recalled from 
the mind and become conscious through neural activity in different association cortical regions.

The fourth enigma is the sense of agency - the sense of self-awareness and knowing that one is the agent of one's actions, feelings, and thoughts. Indeed the principles of agency and moral responsibility are assumptions on which much of human jurisprudence is based. Yet no "center of selfhood" has been found in the brain, and many neuroscientists point to experiments that appear to show that "free will" is illusory because a person's brain appears to "decide" long before the person becomes aware of deciding.

In the present theory, the sense of agency is the sense a person has of being an autonomous mind entity. The mind is the individuality, the being of the person. The mind entity initiates all volitional actions, to move, to direct attention, to think, to speak and so on, by initiating neural activity in the brain. The awareness of a decision arises when the electrical activity associated with deciding appears in the cortex.

\section{Localized or "Non-Local" Consciousness?}

In this view, the fundamental aspect of the mind is the localized individuality or being-ness of the person. In contrast, the idea of "nonlocal consciousness" is derived by extrapolation from quantum physical phenomena that exhibit "non-local" aspects, such as entanglement of particles with instantaneous communication across wide distances and wave-particles that appear to spread over a wide area and interfere with themselves. Proponents of this idea (for example, van Lommel, 2010) extrapolate these physical phenomena to explain NDE phenomena that appear to transcend space and time, such as veridical perceptions in distant locations, previously unknown information from transcendent sources, life reviews, precognitive life previews, and similar paranormal aspects of NDEs. The resulting hypothesis is that consciousness itself is "non-local," meaning beyond or independent of space and time. The suggestion is that during an NDE and at death, the person's mind spreads out, exists everywhere, and encompasses all past and future time.

Our problem with this theory is that it just doesn't fit the phenomenology of NDEs. The localized aspect of the mind manifests throughout the NDE in the persistence of the NDEr's self-conscious awareness with a particular location in space and associated perceptual perspective. The mind appears to be truly individualized rather than being an 
aspect of a greater, non-local "source." Even in the most transcendent experiences in an NDE-for example, a sense of merging with the oneness of all existence-self-awareness and the formation of individual memories are still present. Throughout the NDE, the NDEr retains an individualized perspective, memories, and self-identity. Deceased persons and transcendent, spiritual beings whom the NDEr encounters also display localized, individual natures.

In our view, the individuality of the mind is fundamental and the transcendent or non-local aspects experienced in NDEs are properties of the mind. So a better model and explanation of consciousness should be derived from the phenomenology of the transcendent mind itself as evidenced in NDEs and not from the extrapolation of mysterious-sounding quantum phenomena. Based on phenomena reported in NDEs, consciousness is fundamentally localized in conscious nonmaterial beings who exist in a transmaterial reality that transcends physical space and time. The transmaterial realm subsumes physical reality and has its own phenomenology and laws. Physical reality is derived from the higher transmaterial reality and has the constraints of physical space and time. In earthly incarnations, human beings are both spiritual beings and physical beings, constrained in a physical body in physical space and time, but their essential nature is as individualized, nonmaterial beings. From the physical perspective, this view is dualistic, but from the transcendent perspective, it is monistic.

\section{Hope for a Physical Theory or Promissory Materialism?}

Mitchell-Yellin and Fischer held out hope that an empirical, controlled investigation-presumably such as the AWARE study (Parnia et al., 2014) — would definitively answer whether patients can have accurate AVPs during cardiac arrest. A positive instance of verified veridical perception of a hidden visual target during documented brain inactivity would confirm the hypothesis that consciousness can separate from the physical body and operate independent of brain function. However, the likelihood that such a case will be documented is very small, given that only one case out of more than 2,000 cardiac arrests occurred in the first four years of the AWARE study whereby auditory and visual perceptions were remembered that could be checked. Unfortunately, that NDE occurred in a location of the hospital where no visual target 
had been placed. Nevertheless, veridical perceptions of the equipment and people attending the patient in this NDE were verified.

The failure of any NDErs to notice and accurately describe the hidden target does not disprove the mind-separation hypothesis, because the experimental set up cannot control for where the NDEr directs one's attention. However, any verified veridical perceptions during cardiac arrest are confirmatory of the mind-separation hypothesis, particularly purely visual perceptions that were out of the patient's physical line of sight and of an unusual or idiosyncratic nature (Mays \& Mays, 2009). There are several NDE cases like this, for example, a 1985 quarter located on an 8-foot high cardiac monitor or the 12-digit serial number located on top of a 7 -foot high ventilator machine, mentioned earlier.

In their critique of von Lommel's theory, Mitchell-Yellin and Fischer's strongest argument seems to be that a complete physical explanation of NDEs is possible, "given the possibility of progress of our scientific understanding of the relevant phenomena" (p. 173). Moreover, they said that it is quite reasonable to expect significant progress in the future, particularly given "the nascent state of the relevant sciences (e.g., neuroscience)" (p. 161).

This argument is what Karl Popper called promissory materialism (Popper \& Eccles, 1977, pp. 96-98). For Popper, promissory materialism is a very weak argument and is actually meaningless because it can't be used before the fact to refute any non-physical theory. If the evidence for a complete physical explanation of NDEs finally comes to light through neuroscience, that evidence can be used to refute the non-physical theory. What is more likely to happen, however, is that neuroscience will correct and refine the non-physical theory-if the theory has explanatory power-through the normal process of scientific inquiry. For example, neuroscience could refine the non-physical theory by providing details and empirical evidence of how the nonmaterial mind interfaces with the brain.

\section{Conclusion}

The recent study by Charland-Verville et al. (2014) more clearly showed that proximity to death and fear of imminent death are not the only factors that can trigger an NDE and, indeed, that NDEs appear to happen spontaneously in completely normal, non-life-threatening conditions, such as sleep and meditation. The oft-cited association of NDEs with proximity to death or the fear of imminent death is called 
into question, as is the idea that closer proximity to death generally results in a "deeper" or more meaningful NDE.

In one sense, all NDEs can really be considered "near-death" even if the person remains physically sound during the experience. In the present view, death involves the permanent separation of the mind from the body. In an NDE, the mind separates temporarily from the body, and, thus, all NDErs experience a "taste of death" in their experiences (Janice Holden, personal communication, 2015).

There is now greater confidence that NDE phenomena are completely independent of physiological and psychological causes and represent a common state of consciousness, regardless of the antecedent situation. NDEs appear to be triggered by a number of different precipitating events ranging from acute physiological conditions; to fear of dying; to stress, anxiety and depression; and to spontaneous occurrences such as during sleep or meditation.

The commonality of NDE phenomena in a wide range of physiological and psychological conditions suggests that there is a common proximate cause of the experience. The common proximate cause could be physical, for example, reduced control of sensory processes, a common brain circuit, or a psychotropic agent affecting particular neural receptors. Or the proximate cause could be non-physical, for example, the separation of the nonmaterial mind from the physical body. In either case, the explanation needs to account for essentially all aspects of all NDEs.

If NDEs result from a common physical proximate cause, then there should be corresponding physiological markers-elevated neurotransmitters or characteristic EEG activity in specific brain regions, for example-such that there would be neurological correlates of NDEs that could be detected and diagnosed. A common physical mechanism may also explain why NDEs occur in fewer than $20 \%$ of cardiac arrest survivors. So far, physical explanations of NDEs appear to be incomplete, with significant explanatory gaps, most notably in explaining verified cases of apparently non-physical veridical perceptions (AVPs) in a variety of situations.

If NDEs result from a common non-physical proximate cause, then there should be further cases of AVPs that are documented and thoroughly investigated, and empirical evidence of interaction between the nonmaterial mind and the brain should be demonstrated. In addition, the non-physical hypothesis should provide greater explanatory power for a variety of neurological phenomena such as memory formation 
and recall. So far, non-physical explanations of NDEs appear to have greater explanatory power, including for various general enigmas of consciousness. However, they posit entities and mechanisms that transcend the currently accepted physicalist paradigm.

In our view, there will continue to be these two parallel explanations for NDEs until one is clearly accepted. Acceptance will develop based on (a) the completeness of the explanation in accounting for all aspects of all NDEs, (b) the empirical evidence, and (c) the explanatory power not only of NDEs but of other phenomena as well. The argument that sufficient progress of neuroscientific understanding will ultimately provide a complete physical explanation of NDEs isn't valid before the fact. The question of which explanation is correct will be resolved only with further scientific investigation.

\section{References}

Arnette, J. K. (1992). On the mind/body problem: The theory of essence. Journal of Near-Death Studies, 11, 5-18.

Arnette, J. K. (1995). The theory of essence. II. An electromagnetic-quantum mechanical model of interactionism. Journal of Near-Death Studies, 14, 77-99.

Arnette, J. K. (1999). The theory of essence. III: Neuroanatomical and neurophysiological aspects of interactionism. Journal of Near-Death Studies, 18, 73-101.

Bayne, T., \& Chalmers, D. J. (2003). What is the unity of consciousness? In A. Cleeremans (Ed.), The unity of consciousness: Binding, integration and dissociation (pp. 23-58). Oxford, England: Oxford University Press. doi:10.1093/ acprof:oso/9780198508571.003.0002

Carter, C. (2010). Science and the near-death experience: How consciousness survives death. Rochester, VT: Inner Traditions.

Chalmers, D. J. (1996). The conscious mind: In search of a fundamental theory. New York, NY: Oxford University Press.

Chalmers, D. J. (2010). The character of consciousness. New York, NY: Oxford University Press. doi:10.1093/acprof:oso/9780195311105.001.0001

Charland-Verville, V., Jourdan, J.-P., Thonnard, M., Ledoux, D., Donneau, A.-F., Quertemont, E., \& Laureys, S. (2014). Near-death experiences in non-lifethreatening events and coma of different etiologies. Frontiers in Human Neuroscience, 8, 203. doi:10.3389/fnhum.2014.00203

Facco, E., \& Agrillo, C. (2012). Near-death-like experiences without life-threatening conditions or brain disorders: A hypothesis from a case report. Frontiers in Psychology, 3, 490. doi:10.3389/fpsyg.2012.00490

Gabbard, G. O., \& Twemlow, S. W. (1984). With the eyes of the mind: An empirical analysis of out-of-body states. New York, NY: Praeger.

Greyson, B. (1983). The near-death experience scale: Construction, reliability, and validity. Journal of Nervous and Mental Disease, 171, 369-375. doi:10.1097/00005053-198306000-00007 
Greyson, B. (2000). Dissociation in people who have near-death experiences: Out of their bodies or out of their minds? The Lancet, 355, 460-463. doi:10.1016/ S0140-6736(00)82013-9

Greyson, B. (2010a). Implications of near-death experiences for a postmaterialist psychology. Psychology of Religion and Spirituality, 2(1), 37-45. doi:10.1037/ a0018548

Greyson, B. (2010b). Seeing dead people not known to have died: "Peak in Darien" experiences. Anthropology and Humanism, 35(2), 159-171. doi:10.1111/ j.1548-1409.2010.01064.x

Holden, J. M. (2009). Veridical perception in near-death experiences. In J. M. Holden, B. Greyson, \& D. James (Eds.), The handbook of near-death experiences: Thirty years of investigation (pp. 185-211). Santa Barbara, CA: Praeger/ ABC-CLIO.

Khanna, S., \& Greyson, B. (2014). Near-death experiences and spiritual wellbeing. Journal of Religion and Health, 53(6), 1605-1615. doi:10.1007/ s10943-013-9723-0

Libet, B. (2004). Mind time: The temporal factor in consciousness. Cambridge, MA: Harvard University Press.

Mays, R. G., \& Mays, S. B. (2008). The phenomenology of the self-conscious mind. Journal of Near-Death Studies, 27(1), 5-45.

Mays, R. G., \& Mays, S. B. (2009). On the scope of analysis for the AWARE study. Journal of Near-Death Studies, 27(3), 195-201.

Mays, R. G., \& Mays, S. B. (2011). A theory of mind and brain that solves the 'hard problem' of consciousness. Paper presented at the annual conference of the International Association for Near-Death Studies (IANDS), Durham, NC. Retrieved from http://selfconsciousmind.com/papers.html\#theory-article

Mitchell-Yellin, B., \& Fischer, J. M. (2014). The near-death experience argument against physicalism: A critique. Journal of Consciousness Studies, 21(7-8), 158-183.

Owens, J. E., Cook, E. W., \& Stevenson, I. (1990). Features of "near-death experience" in relation to whether or not patients were near death. The Lancet, 336, 1175-1177. doi:10.1016/0140-6736(90)92780-L

Parnia, S., Spearpoint, K., de Vos, G., Fenwick, P., Goldberg, D., Yang, J., . . . \& Schoenfeld, E. R. (2014). AWARE-AWAreness during REsuscitation-A prospective study. Resuscitation, 85(12), 1799-1805. doi:10.1016/j.resuscitation .2014.09.004

Popper, K. R., \& Eccles, J. C. (1977). The self and its brain: An argument for interactionism. London, England, UK: Routledge. doi:10.1007/978-3-642-61891-8

Raichle, M. E., \& Snyder, A. Z. (2007). A default mode of brain function: A brief history of an evolving idea. Neuroimage, 37(4), 1083-1090. doi:10.1016/j.neuro image.2007.02.041

Rivas, T., \& Smit, R. H. (2013). A near-death experience with veridical perception described by a famous heart surgeon and confirmed by his assistant surgeon. Journal of Near-Death Studies, 31, 179-186.

Rivas, T., Dirven, A., \& Smit, R. H. (2013). Wat een stervend brein niet kan: aanwijzingen voor parapsychologische verschijnselen rond bijna-doodervaringen; de harde kern van bevestigde casussen [What a dying brain can't do: Evidence of parapsychological phenomena connected with near-death experiences-The main confirmed cases]. Leeuwarden, Netherlands: Elikser B. V. 
Smith, S. M., Fox, P. T., Miller, K. L., Glahn, D. C., Fox, P. M., Mackay, C. E., . . Beckmann, C. F. (2009). Correspondence of the brain's functional architecture during activation and rest. Proceedings of the National Academy of Sciences, 106(31), 13040-13045. doi:10.1073/pnas.0905267106

Thibaut, A., Bruno, M. A., Chatelle, C., Gosseries, O., Vanhaudenhuyse, A., Demertzi, A., . . Laureys, S. (2012). Metabolic activity in external and internal awareness networks in severely brain-damaged patients. Journal of Rehabilitation Medicine, 44(6), 487-494. doi:10.2340/16501977-0940

Thonnard, M., Charland-Verville, V., Brédart, S., Dehon, H., Ledoux, D., Laureys, S. \& Vanhaudenhuyse, A. (2013). Characteristics of near-death experiences memories as compared to real and imagined events memories. PLoS ONE, 8(3): e57620. doi:10.1371/journal.pone.0057620

van Lommel, P. (2010). Consciousness beyond life: The science of the near-death experience. New York, NY: Harper-Collins.

van Lommel, P. (2013). Non-local consciousness: A concept based on scientific research on near-death experiences during cardiac arrest. Journal of Consciousness Studies, 20(1-2), 7-48.

Zingrone, N. L., \& Alvarado, C. S. (2009). Pleasurable Western adult near-death experiences: Features, circumstances, and incidence. In J. M. Holden, B. Greyson, \& D. James (Eds.), The handbook of near-death experiences: Thirty years of investigation (pp. 17-40). Santa Barbara, CA: Praeger/ABC-CLIO. 